\title{
Numerical Solutions for Some Free Boundary Value Problems Occuring in Planar Fluid Dynamics
}

\author{
R.P. GILBERT ${ }^{1)}$ and WEN GUO-CHUN
}

In the paper [1], we consider the solvability of some free boundary value problems occuring in planar fluid dynamics. The object of the present paper is to present numerical methods for - solving free boundary problems. It was shown in [1] that such free boundary.problems may be transformed into a mixed boundary value problem for a linear or nonlinear elliptic complex equations, which in turn, might be reformulated into a conformal mapping or quasiconformal mapping from a general domain onto some canonical domain. In this paper we direct our discussion to the numerical solution of mixed boundary value problems for generalized Beltrami equations.

Key words: Numerical solutions, free boundary problems

AMS subject classification: 35 r $35,65 \mathrm{~N} 30$

\section{\$1 Numerical solutions of mixed boundary value problems}

Let $D$ be a $(N+1)$-connected domain with the boundary $\Gamma=\bigcup_{j=0}^{N} \Gamma_{j}$, where the sets $\Gamma_{j}=\{z: \mid z-$ $\left.z_{j} \mid=\gamma_{j}\right\}(j=1, \ldots, N)$ are situated inside $\Gamma_{0}=\{z:|z|=1\}$ and $z=0 \in D$. We consider, in $D$, the nonlinear elliptic complex equation

$$
w_{\bar{z}}=F\left(z, w, w_{z}\right), \quad F=Q_{1} w_{z}+Q_{2} \bar{w}_{\bar{z}}+A_{1} w+A_{2} \bar{w}+A_{3},
$$

in which $Q_{j}=Q_{j}\left(z, w, w_{z}\right)(j=1,2)$ and $A_{j}=A_{j}(z, w)(j=1,2,3)$. Suppose that the complex equation (1.1) satisfies condition

(C) 1. The functions $Q_{j}(z, w, U)(j=1,2)$ and $A_{j}(z, w)(j=1,2,3)$ are measurable in $z \in D$ for all continuous functions $w$ and all measurable functions $U$ in $D$ and satisfy the bounds

$$
L_{p}\left[A_{j}(z, w(z)), \bar{D}\right] \leq k_{\mathrm{o}}<\infty \quad(j=1,2,3)
$$

where $p(2<p<\infty)$ and $k_{0}\left(0 \leq k_{0}<\infty\right)$ are real constants.

2. The complex equation (1.1) satisfies the uniform ellipticity condition

$$
\left|F\left(z, w, U_{1}\right)-F\left(z, w, U_{2}\right)\right| \leq q_{0}\left|U_{1}-U_{2}\right| \text { for a.e. } z \in D \text { and } w, U_{1}, U_{2} \in \mathbb{C} \text {, }
$$

where $q_{0}\left(0 \leq q_{0}<1\right)$ is a constant.

3. The functions $Q_{j}(z, w, U)(j=1,2)$ and $A_{j}(z, w)(j=1,2,3)$ are continuous for $w \in \mathbb{C}$ for

1) Supported in Part by the National Science Foundation through grant INT - 9011085. 
a.e. point $z \in D$, and $F(z, w, U)$ satisfies the condition

$$
\left|F\left(z, w_{1}, U\right)-F\left(z, w_{2}, U\right)\right| \leq R(z)\left|w_{1}-w_{2}\right| \text { for } w_{1}, w_{2}, U \in \mathbb{C},
$$

where $L_{p}(R(z), \bar{D}) \leq k_{0}$.

4. The function

$$
G(w, V)=\left[w_{\bar{z}}-F\left(z, w ; w_{z}\right)\right]\left[\bar{V}_{z}-\overline{F_{w} V}-\overline{F_{\bar{w}} \bar{V}}-\overline{F_{w_{z}} V_{z}}-\overline{F_{\bar{w}} V_{\bar{z}}}\right]
$$

satisfies the condition

$$
G\left(w_{1}, w_{1}-w_{2}\right)-G\left(w_{2}, w_{1}-w_{2}\right)=\left|w_{1 \bar{z}}-F\left(z, w_{1}, w_{1 z}\right)-\left(w_{2 \bar{z}}-F\left(z, w_{2}, w_{2 z}\right)\right)\right|^{2} .
$$

In particular, if the complex equation (1.1) is linear, i.e. $Q_{j}=Q_{j}(z)(j=1,2)$ and $A_{j}=A_{j}(z)(j=$ $1,2,3)$, then the conditions (1.2) - (1.4) obviously hold.

Problem A: The so-called mixed boundary value problem for (1.1) is to find a bounded solution $w \in D^{*}$ satisfying the boundary condition

$$
\operatorname{Re}[\overline{\lambda(t)}(t)]=r(t)+h(t)\left(t \in \Gamma^{*}\right)
$$

in which $|\lambda(t)|=1, \lambda$ and $r$ posses discontinuities of the first kind at the points $t_{1}, \ldots, t_{m}, D^{*}=$ $\vec{D} \backslash\left\{t_{1}, \ldots, t_{m}\right\}$ and $\Gamma^{*}=\Gamma \backslash\left\{t_{1}, \ldots, t_{m}\right\}$ consists of the arcs $\Gamma^{1}, \ldots, \Gamma^{n}$ with end points from the set $\left\{t_{1}, \ldots, t_{m}\right\}$, where the $t_{1}, \ldots, t_{m}$ are arranged in with a positive orientation. If $\lambda$ is continuous on $\Gamma_{j}(0 \leq j \leq N)$, then we add an arbitrarily chosen point $t^{*} \in \Gamma_{j}$ to the set $\left\{t_{1}, \ldots, t_{m}\right\}$ and relabel this set as $\left\{t_{1}, \ldots, t_{m}\right\}$. Moreover $\lambda$ and $r$ satisfy the conditions

$$
\begin{aligned}
& \lambda(t)=\mathrm{e}^{\mathrm{i} \varphi_{j}}, \varphi_{j}=\beta_{j} \pi, 0 \leq \beta_{j}<2 \\
& r(t)=r_{j}(t), C_{\alpha}\left[r_{j}(t), \Gamma^{j}\right] \leq 1<\infty, j=1, \ldots, n
\end{aligned}
$$

where $1 / 2<\alpha<1$ and $B_{j}, l$ are nonnegative constants. Besides,

$$
h(t)= \begin{cases}h_{j}, t \in \Gamma_{j}, j=1, \ldots, N & \\ 0, t \in \Gamma_{j}, j=0 & \text { for } x=0 \\ h_{j}, t \in \Gamma_{j}, j=0,1, \ldots, N & \text { for } x=-1\end{cases}
$$

where $h_{j}(j=0,1, \ldots, N)$ are unknown constants to be determined appropriately, and $x=0$ or $x=$ -1 is the index of Problem A (see [1,2]). If $x=0$, we may require that the solution $w$ satisfies the point condition

$$
\left.\operatorname{Im}\left[\overline{\lambda\left(a_{j}\right.}\right) w\left(a_{j}\right)\right]=b_{j}, j \in\{j\}=1,
$$

in where $a_{1}\left(\neq t_{k}\right) \in \Gamma_{0}, b_{1}$ is a real constant with the condition $\left|b_{1}\right| \leq 1$. The mixed boundary value problem in [1] can be reduced to the Problem $A$ above.

By $H^{1}(D)$ we denote the Sobolev space $H^{1}(D)=W_{2}^{1}(D)=\left\{w \mid w, w_{\bar{z}}, w_{z} \in L_{2}(D)\right.$, and denote by Problem $B$ the extremal problem for the functional $I=I(u)$, i.e. we find a bounded function $w \in H^{1}(D) \cap \cap C\left(D^{*}\right)$, such that

$$
I(w)=\min _{u \in H^{1}(D) \ln C\left(D^{*}\right)} I(u)
$$


where

$$
\begin{aligned}
I(u)= & \iint_{D}\left|u_{z}-F\left(z, u, u_{z}\right)\right|^{2} d \sigma_{z} \\
& +\int_{\Gamma}|\operatorname{Re} \overline{\lambda(t)} u(t)-r(t)-h(t)|^{2} d s+\sum_{j \in\{j\}}\left(\operatorname{Im} \overline{\lambda\left(a_{j}\right)} u\left(a_{j}\right)-b_{j}\right)^{2} .
\end{aligned}
$$

It is easy to see that the unknown constants $h_{j}=\operatorname{Re} \overline{\lambda\left(a_{j}\right)} u\left(a_{j}\right)-r\left(a_{j}\right)$, in which $a_{j}\left(\neq t_{k}\right)$ is a point on $\Gamma_{j}(j=0,1,2, \ldots, N)$, which we sometimes denote as $h(t)=h\left(t, u^{*}\right)$. In the formula (1.7), the double integral is over $D_{\varepsilon}=D \backslash \sum_{j=1}^{m}\left\{\left|z-t_{j}\right|<\varepsilon\right\}$, where $\varepsilon$ is an arbitrary small positive number.

Theorem 1.1: The function $w$ is a solution of Problem $A$ if and only if it is a solution of Problem B.

Proof: Substitute the solution $w$ of Problem A for $u$ in (1.7). Obviously $I(w)=0$, which shows that $w$ is a solution of Problem B.

Conversely, let $w$ be a solution of Problem B. Then it is clear that $w$ satisfies the first order complex equation (1.1) for almost every point $z \in D$, the boundary condition (1.5) on $\Gamma^{*}$ and the point condition (1.6). If we can prove that $w \in W_{p_{0}}^{1}\left(D_{\varepsilon}\right), 2<p_{0}<p$, then $w$ is also a solution of Problem A. According to [3: Theorem 2.1] and [4: Chap. 4/Theorem 1.3], $w$ can be expressed as $w(z)=\Phi[\zeta(z)] \mathrm{e}^{\varphi(z)}+\Psi(z)$, where $\zeta, \varphi, \Psi \in W_{P_{0}}^{1}(D), w \in W_{P_{0}}^{1}[\zeta(D)]$ and $\Phi$ is analytic in $\zeta(D)$. lt can be shown that $w \in W_{P_{0}}^{1}\left(D_{\varepsilon}\right)$

We use a regular triangular net to subdivide the domain $D$, such that the diameter of each triangular unit $\Delta_{j}$ is not greater than a positive constant $h$. Denote the vertices by $z_{1}, \ldots, z_{n}$ so that $a_{j} \in\left\{z_{1}, \ldots, z_{n}\right\}(j=0,1,2, \ldots, N)$. Moreover, we insist that $t_{k},\left\{z_{1}, \ldots, z_{n}\right\}(k=1,2, \ldots, m)$. We introduce the space $E$ of complex linear splines,

$$
E=\left\{v \mid v \text { is linear in } z, \bar{z} \text { on each } \Delta_{j} \text { and continuous at } z_{j} \in D\right\} \text {. }
$$

It is not difficult to see that $E \subset H^{1}(D) \cap C\left(D^{*}\right)$. We use $\varphi_{k} \in E$, where $\varphi_{k}\left(z_{j}\right)=1$ for $j=k$ and $\varphi_{k}\left(z_{j}\right)=0$ for $j \neq k(j, k=1, \ldots, n)$. A basis for the space $E$ consists of the function set $\left\{\varphi_{1}, \ldots\right.$, $\left.\varphi_{n}, i \varphi_{2}, \ldots, i \varphi_{n}\right\}$, where $i$ is the imaginary unit.

Theorem 1.2: Suppose that $R \in L^{\infty}(\bar{D})$ in Condition $C$. Let $w$ be an exact solution of Proble $m B$ and $\hat{w} \in E$ be a linear interpolation function of $w$. Then Problem $B$ has a solution.

Proof: We first prove $I(\hat{w}) \leq M_{1} h^{2}$, where $M_{1}$ is constant. In fact, we have that

$$
\begin{aligned}
J(\hat{w})= & \iint_{D}\left|\hat{w}_{\bar{z}}-F\left(z, \hat{w}, \hat{w}_{z}\right)\right|^{2} d \sigma_{z} \\
& \left.+\int_{\Gamma}|\operatorname{Re} \overline{\lambda(t)} \hat{w}(t)-r(t)-h(t)|^{2} d s+\sum_{j c\{j\}}\left(\operatorname{Im} \overline{\lambda\left(a_{j}\right.}\right) \hat{w}\left(a_{j}\right)-b_{j}\right)^{2} \\
= & \iint_{D}\left|\hat{w}_{z}-F\left(z, \hat{w}, \hat{w}_{z}\right)\right|^{2} d \sigma_{z}+\int_{\Gamma}|\operatorname{Re} \overline{\lambda(t)}(\hat{w}(t)-w(t))|^{2} d s .
\end{aligned}
$$

Noting that $w \in H^{2}(D) \cap C\left(D^{*}\right)$, it may be extended to a function $w^{*} \in H^{1}\left(\mathbb{R}^{2}\right) \cap C\left(D^{*}\right)$, i.e. $w^{\mathcal{C}}(z)=w(z)$, for $z \in D$, and $\left\|w^{*}\right\|_{H^{1}\left(\mathbb{R}^{2}\right)} \leq M_{2}\|w\|_{H^{1}(D)}$ where $M_{2}$ is a constant. From the trace 
theorem, it follows that

$$
\begin{aligned}
\int_{\Gamma}|\operatorname{Re} \overline{\lambda(t)}(\hat{w}(t)-w(t))|^{2} d s & \leq M_{3}\|\hat{w}-w\|_{H^{2}(D)}^{2} \leq M_{3}\left\|\hat{w}-w^{*}\right\|_{H^{1}(D)}^{2} \\
& \leq M_{3} h^{2}\left\|_{W^{*}}\right\|_{H^{1}(D)}^{2} \leq M_{3} h^{2}\left\|_{W}\right\|_{H^{1}(D)}^{2} \leq M_{4} h^{2},
\end{aligned}
$$

where $M_{3}, M_{4}$ are constants. Moreover,

$$
\begin{aligned}
& \iint_{D}\left|\hat{w}_{\bar{z}}-F\left(z, \hat{w}, \hat{w}_{z}\right)\right|^{2} d \sigma_{z} \\
& \quad=\iint_{D}\left|(\hat{w}-w)_{z}-\left(F\left(z, \hat{w}^{\prime}, \hat{w}_{z}\right)-F\left(z, w, w_{z}\right)\right)\right|^{2} d \sigma_{z} \\
& \quad s \iint_{D}\left|(\hat{w}-w)_{\bar{z}}-\left(F\left(z, \hat{w}, \hat{w}_{z}\right)-F\left(z, \hat{w}, w_{z}\right)\right)-\left(F\left(z, \hat{w}, w_{z}\right)-F\left(z, w, w_{z}\right)\right)\right|^{2} d \sigma_{z} \\
& \quad \leq \iint_{D}\left((\hat{w}-w)_{\bar{z}}+q_{0}\left(w_{z}-\hat{w}_{\bar{z}}\right)+|R(z)||\hat{w}-w|^{2}\right) d \sigma_{z} . \\
& \quad \leq M_{s}\|\hat{w}-w\|_{H^{2}(D)}^{2} \leq M_{0} h^{2},
\end{aligned}
$$

in which $M_{5}, M_{6}$ are constants.

Secondly, using reductio ad absurdum, we can prove that there exists a positive constant $M_{7}(h)$, such that

$$
\begin{aligned}
& \iint_{D}\left|u_{\bar{z}}-F\left(z, u, u_{z}\right)+F(z, 0,0)\right|^{2} d \sigma_{z} \\
& \quad+\sum_{j=0}^{N} \int_{\Gamma_{j}}\left|\operatorname{Re} \overline{\lambda(t)} u(t)-\operatorname{Re} \overline{\lambda\left(a_{j}\right)} u\left(a_{j}\right)\right|^{2} d s+\sum_{j c\langle j\}} \operatorname{Im} \overline{\lambda\left(a_{j}\right)} u\left(a_{j}\right) \\
& \quad=I_{0}(u) \geq M_{7}(h)\|u\|_{H^{1}(D) \cap C\left(D^{*}\right)}^{2}
\end{aligned}
$$

where $\|u\|_{H^{1}(D) \cap C\left(D^{*}\right)}=\|u\|_{H^{1}(D)}^{2}+C\left(u, D^{*}\right)$, and if $x=0, \operatorname{Re} \overline{\lambda\left(a_{0}\right)} u\left(a_{0}\right)$ is represented by 0 . Obviously,

$$
\min _{u \in E} l_{0}(u)=\min _{u \in E, I(u) \leq M_{1} h^{2}} I_{0}(u) .
$$

It remains to be shown that (1.8) possesses a minimum. As a matter of fact,

$$
\begin{aligned}
I_{0}(u)= & \iint_{D}\left|u_{z}-F\left(z, u, u_{z}\right)+F(z, 0,0)\right|^{2} d \sigma \\
& +\sum_{j=0}^{N} \int_{\Gamma_{j}}\left|\left(\operatorname{Re} \overline{\lambda(t)} u(t)-r(t)-\operatorname{Re} \overline{\lambda\left(a_{j}\right)} u\left(a_{j}\right)+r\left(a_{j}\right)\right)+r(t)-r\left(a_{j}\right)\right| d s \\
& +\sum_{j<\{j\}}\left(\left(\operatorname{Im} \overline{\lambda\left(a_{j}\right)} u\left(a_{j}\right)-b_{j}\right)+b_{j}\right)^{2} \\
& \leq 2\left(M_{i} h^{2}+\iint_{D}|F(z, 0,0)|^{2} d \sigma_{z}+\sum_{j=0}^{N} \int_{\Gamma_{j}}\left|r(t)-r\left(a_{j}\right)\right|^{2} d s+\sum_{j c\{j\}}\left|b_{j}\right|^{2}\right)=M_{8}(h) .
\end{aligned}
$$


Consequently, $\max _{z \in D^{-}}|u| \leq M_{q}(h)<\infty$. We consider next $u=\sum_{i=1}^{n} c_{i} q_{i}+\sum_{j=n+1}^{2 n} c_{j}\left(i q_{i}\right)$; we notice that $\max _{1 \leq i \leq 2 n}\left|c_{i}\right| \leq M_{9}(h)$. Hence there exists a $w_{h} \in E$, such that $I\left(w_{h}\right)=\min _{u \in E} I(u)$

If $u=w_{h}$, the variation of $I(u)$ is equal to zero, i.e.

$$
\begin{aligned}
\delta I\left(w_{h}\right)= & 2 \operatorname{Re} \iint_{D}\left(w_{h \bar{z}}-F\left(z, w_{h}, w_{h z}\right)\right)\left(\ddot{u}_{z}-F_{w_{h}} u-F_{\overline{w_{h}}} \bar{u}-F_{w_{h z}} u_{z}-F_{\bar{w}_{h} \bar{z}} \bar{u}_{\bar{z}}\right) d \sigma_{z} \\
& +\sum_{j=0}^{N} \int_{\Gamma_{j}}\left(\operatorname{Re} \overline{\lambda(t)} w_{h}(t)-r(t)-\operatorname{Re} \overline{\lambda\left(a_{j}\right)} w_{h}\left(a_{j}\right)+r\left(a_{j}\right)\right)\left(\operatorname{Re} \overline{\lambda(t)} u(t)-\operatorname{Re} \overline{\lambda\left(a_{j}\right)} u\left(a_{j}\right)\right) d s \\
& +\sum_{j \in\{j\}}\left(\operatorname{Im} \overline{\lambda\left(a_{j}\right)} w_{h}\left(a_{j}\right)-b_{j}\right) \operatorname{Im} \overline{\lambda\left(a_{j}\right)} u\left(a_{j}\right) \\
= & a\left(w_{h}, u\right)=0 .
\end{aligned}
$$

Variational Problem C: Find a complex function $w_{h} \in E$, such that $a\left(w_{b}, u\right)=0$ for all $u \in E$.

Theorem 1.3: A necessary and sufficient condition that $w_{h}$ is a solution of Problem $B$, is that it is a solution of Problem $C$.

Proof: The necessity of the hypothesis is obvious. Now suppose $w_{h}$ is a solution of Problem C. By Theorem 1.1 and by the necessity put of this theorem, $w_{h}$ is a solution of Problem B. If the solution of Problem $C$ is unique, then sufficiency is proved. Suppose to the contrary that $w_{1 h}, w_{2 h}$ are two solutions of Problem C. Since $a\left(w_{1 h}, u\right)=0, a\left(w_{2 h}, u\right)=0$, for all $u \in E$, it follows that $a\left(w_{1 h}, u\right)-a\left(w_{2 h}, u\right)=0$. Setting $w_{h}^{*}=w_{1 h}-w_{2 h}$ and using Condition $\mathrm{C}$, it can be seen that

$$
\begin{aligned}
& \iint_{D} \mid w_{1 h \bar{z}}-F\left(z, w_{1 h}, w_{1 h z}\right)-\left(w_{2 h \bar{z}}-\left.F\left(z, w_{2 h}, w_{2 h z}\right)\right|^{2} d \sigma_{z}\right. \\
& \quad+\sum_{j=0}^{N} \int_{\Gamma_{j}}\left|\operatorname{Re} \overline{\lambda(t)}\left(w_{1 h}(t)-w_{2 h}(t)\right)-\operatorname{Re} \overline{\lambda\left(a_{j}\right)}\left(w_{1 h}\left(a_{j}\right)-w_{2 h}\left(a_{j}\right)\right)\right|^{2} d s \\
& \quad+\sum_{j \in\{j\}}\left|\operatorname{lm} \overline{\lambda\left(a_{j}\right)}\left(w_{1 h}\left(a_{j}\right)-w_{2 h}\left(a_{j}\right)\right)\right|^{2}=0,
\end{aligned}
$$

and $w_{h}^{*}$ is a bounded solution of the following boundary value problem:

$$
\begin{array}{ll}
w_{h \bar{z}}^{*}=Q(z) w_{h z}^{*}+A(z) w_{h}^{*} & \left(|Q(z)| \leq q_{0}<1, A \in L_{p}(\bar{D})\right) \\
\left.\operatorname{Re}\left(\overline{\lambda(t)} w_{h}^{*}(t)\right)=\operatorname{Re}\left(\overline{\lambda\left(a_{j}\right.}\right) w_{h}\left(a_{j}\right)\right) & \left(a_{j} \in \Gamma_{j}, 0 \leq j \leq N\right) \\
\operatorname{Im}\left(\overline{\lambda\left(a_{j}\right)}\left(w_{h}^{*}\left(a_{j}\right)\right)=0\right. & (j=1, \text { for } x=0) .
\end{array}
$$

According to the uniqueness theorem, we conclude $w_{h}^{*}=0$. i.e. $w_{1 h}=w_{2 h}$

Similar arguments can be used to obtain error estimates. 


\section{$\$ 2$ Numerical method for some quasiconformal mappings}

A quasiconformal mapping may be shown to be a homeomorphic solution of an elliptic complex equation of first order

$$
w_{\bar{z}}=F\left(z, w, w_{z}\right), \quad F=Q_{1} w_{z}+Q_{2} \bar{w}_{\bar{z}}=Q w_{z}
$$

defined in the domain $D$, where $O:=Q_{1}(z) w_{z}+Q_{2}(z) \bar{w}_{\bar{z}} / w_{z}$. We assume that (2.1) satisfies Condition $C$ stated as $\$ 1$. In [4: Chap. 3], we proved that there exists a unique solution $w$ of the complex equation (2.1), and that this solution quasiconformally maps the domain $D$ onto some canonical domains. The conformal mappings and quasiconformal mappings may be reduced to Boumdary Value Problem $Q$ for the complex equation (2.1), i.e. we improve the condition

$$
\operatorname{Re}(\overline{\lambda(t)} w(t))=r(t)+h(t), \quad t \in \Gamma,
$$

where

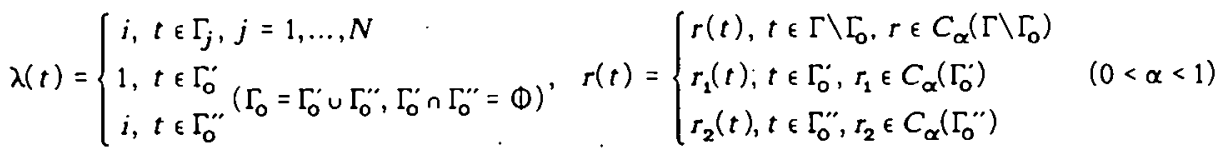

$$
\begin{aligned}
& h(t)= \begin{cases}h_{j}, & t \in \Gamma_{j}, j=1, \ldots, N \\
h_{0}, t \in \Gamma_{0}, \text { if } x=0,\end{cases}
\end{aligned}
$$

in which the $h_{j}(j=1, \ldots, N)$ are unknown constants to be determined, $\Gamma_{0}^{\prime}$ and $\Gamma_{0}^{\prime \prime}$ are some subarcs of $\Gamma_{0}$. Similarly to $\S 1$, the indexes of Problem $Q$ are $x=0$ or $x=-1$. If $x=0$, we take $h_{0}$ $=0$. In some cases, the solutions $w$ of Problem $Q$ are not bounded. Using the method of [2: Chap. 3/Theorem 6.1], Problem $Q$ can be transformed into another boundary value problem. Applying the method mentioned and the representation theorem therein for solutions of the complex equation (2.1), we can be proved that the solution of Problem $Q$ under certain conditions is a homeomorphism from $D$ onto the desired canonical domain.

Next, we construct a homeomorphic solution of (2.1) that quasiconformally maps $D$ onto a strip lying between $\operatorname{Im} w=0, \operatorname{Im} w=1$ and having $N$ level rectilinear slits deleted from in $0<$ $\operatorname{Im} w<1$. In this case, the boundary condition (2.2) of Problem $Q$ may be put in the following form:

$$
\operatorname{Re}(\overline{\lambda(t) w}(t))=r(t)+h(t):=\left\{\begin{array}{cc}
0, & -\pi<\vartheta<0 \\
1, & 0<\vartheta<\pi \\
h_{j}, & t \in \Gamma_{j}, j=1, \ldots, N
\end{array}\left(t=\mathrm{e}^{\mathrm{i} \vartheta} \in \Gamma_{\mathrm{o}}\right)(w(\mathrm{i})=\mathrm{i} \pi), \overline{\lambda(t)}=-\mathrm{i}, \quad t \in \Gamma .\right.
$$

We choose an analytic function $H(z)=1 / \pi\left(\ln \frac{1+z}{1-z}+\mathrm{i} \pi / 2\right)$ such that $H(0)=\mathrm{i} / 2$, which conformally maps $|z|<1$ onto the strip $0<\operatorname{Im} H<1$. Setting $w=\mathrm{i} W+H$, Problem $\mathrm{Q}$ for (1.1) can be reduced to the following boundary value Problem R:

$$
\begin{aligned}
& W_{\bar{z}}=G\left(z, W_{z}\right), G=Q_{1}(z) W_{z}-Q_{2}(z) \bar{W}_{\bar{z}}-\mathrm{i} A(z), A=Q_{1} H^{1}+Q_{2} \bar{H}^{1} \\
& \operatorname{Re} W(t)=R(t)=\left\{\begin{array}{cc}
0, & t \in \Gamma_{0} \\
-\operatorname{Im} H(t)+h(t), t \in \Gamma \backslash(\Gamma \backslash\{0\})
\end{array}(W(\mathrm{i})=0) .\right.
\end{aligned}
$$

We assume that $A \in L_{p}(\bar{D}), p>2$. 
Theorem 2.1: The function $w$ is a quasiconformal mapping for Problem $Q$ if and only if $W$ $=-\mathrm{i}(w-H)$ is a bounded solution of Problem $R$.

Proof: It is clear that if $w$ is a quasiconformal mapping, then $W=-\mathrm{i}(w-H)$ is a bounded solution of Problem R. Conversly, if $W$ is a bounded solution of Problem $R$, then $w=i W+h$ can be expressed as $w(z)=\Phi(\zeta(z))$, where $\zeta$ is a homeomorphism which quasiconformally maps $D$ onto a bounded circular domain $\Omega$ and, $\Phi(\zeta)$ ia an analytic function in the domain $\Omega$, satisfying the boundary condition $\operatorname{Re}(\overline{\lambda(z(\zeta)} \Phi(\zeta))=r(z(\zeta)), \zeta \in \Gamma=\partial \Omega$, where $z(\zeta)$ is the inverse function of $\zeta(z)$. Using the method of [2: Chap. 3, §3] we can prove that $\Phi(\zeta)$ conformally maps the domain $\zeta(D)$ onto the strip domain with the boundary $\operatorname{Im} w=0, \operatorname{Im} w=1$ and $N$ level rectilinear slits lying between $0<\operatorname{Im} w<1$

In order to find the numerical solution of Problem $R$ for the complex equation (2.3), we introduce the extremal Problem $\mathrm{S}$ for $I(U)$ :

$$
I(W)=\min _{u \in H^{1}(D) n C(\bar{D})}\left\{\iint_{D}\left|U_{z}-G\left(z, U_{z}\right)\right|^{2} d \sigma_{z}+\int_{\Gamma}|\operatorname{Re} W(t)-R(t)|^{2} d s+|W(\mathrm{i})|^{2}\right\}
$$

and the variational Problem T:

$$
\begin{aligned}
\partial I(W)= & 2 \operatorname{Re} \iint_{D}\left|W_{\bar{z}}-G\left(z, W_{z}\right)\right|\left|\bar{U}_{z}-\overline{G\left(z, U_{z}\right.}\right| d \sigma_{z} \\
& +2 \int_{\Gamma}|\operatorname{Re} W(z)-R(z, W)||\operatorname{Re} U(z)-R(z, U)| d s+2 \operatorname{Re} W(\mathrm{i}) U(\mathrm{i}),
\end{aligned}
$$

where $R(z, W)=0$ for $t \in \Gamma_{0}$ and $R(z, W)=-\operatorname{Im} H(t)+\operatorname{Re} W\left(a_{j}\right)+\operatorname{Im} H\left(a_{j}\right), j=1, \ldots, N$. Using the finite element method, we can construct a numerical solution $W_{h}$ of Problem $\mathrm{T}$ and Problem $\mathrm{S}$. The function $W_{h}$ is also a numerical solution of Problem $R$. Therefore $w_{h}=i W_{h}+H$ is a numerical solution of Problem $Q$.

\section{REFERENCES}

[1] GILBERT, R.P. and WEN GUO-CHUN: Freo boundary problems occuring in planar fluid dynamics. Nonlin. Anal. Theory Appl. 13 (1989), 285 - 303.

[2] WEN GUO-CHUN: Conformal Mappings and Boundary Value Rroblems. Amer. Math. Soc. 1992.

[3] WEN GUO-CHUN: Numerical solutions for elliptic boundary value problems and quasiconformal mappings. J. Sichuan Norm. Univ. (Nat. Sci.) 4 (1989), 7 - 20.

[4] WEN GUO-CHUN: Linear and Nonlinear Elliptic Complex Equations. Shanghai Sci. Techn. Publ. 1986 (in Chinese).

[5] Ciarler, P.G.: The Finite Element Mothod for Elliptic Problems. Amsterdam: NorthHolland 1978.

Received 10.02.1992

Prof. Dr. Robert P. Gilbert

University of Delaware

Department of Math. Sciences

Nowark, Delaware 19716, USA
Prof. Dr. Wen Guo-chun

Poking University

Department of Mathematics

Beijing 100871. P.R. China 
K. Strehmel (ed.): Numerical Treatment of Differential Equations. Proceedings of the Fifth Seminar "NUMDIFF-5" held in Halle 1989 (Teubner-Texte zur Mathematik: Vol. 121). Stuttgart - Leipzig: B.G. Teubner Verlagsges. 1991; 372 pp.

With the present Volume 121 of the series "Teubner-Texte zur Mathematik", Teubner-Verlag continues the publication of proceedings of the by now traditional NUMDIFF workshops which have been held at the Mortin Luther University of Halle. Wittenberg since 1981. $\wedge s$ can be estimated from both the list of speakers, and the papers presented, NUMDIFF-5 has found considerable resonance among the European mathematical community. The central goal of the workshops, to support the exchange of the latest scientific developments in the broad field of numerical analysis for ordinary differential equations including its relationships to other mathematical disciplines and to applications in modelling real-world processes, is reflected by the papers selected for the proceedings by the editor (and chairman of the NUMDIFF seminars) K. Strehmel.

The first and main part of the proceedings is devoted to the analysis of numerical methods for ordinary differential equations. Initial value problems for algebro-differential equations are discussed in the contributions by Arnold, Griepentrog. Hanke. März, Niepage, Reich, and Roche, who cover almost all aspects of this topic of current research interest. The survey article by Hairer and the paper by Strehmel/Weiner focus on the relation between algebro-differential equations and certain classes of singularly perturbed stiff systems of ordi. nary differential equations. Efficient solvers for stiff problems are dealt with by Auzinger/ Frank/Kirlinger (extrapolation schemes), Kaps/Ostermann und Scholz (methods of Rosen brock type). Houwen addresses parallelization aspects in the implementation of block Runge.Kutta methods, and Hout/Spijker contribute some results on algorithms for delay equations. The papers on numerical methods for boundary value problems for ordinary differential equations are relatively isolated from each other, and cover very different methods and problem classes: shooting algorithms for parameter dependent nonlinear two-point boundary value problems (Hermann/Ullmann) and algebro-differential equations (Lamour), the analysis of the $\tau$-method (Pfeifer/Roos), iterative techniques using a formulation as integral equation (Quinney/Croft), and issues of grid selection in finite difference discretizations (Schmitt/Schild).

The second part of the proceedings is concentrating on the application of numerical techniques for ordinary differential equations to the discretization of partial differential equations. The most typical such problem class, the method of lines for evolution problems, is discussed in the papers by Farago, Grigorief, Hundsdorfer, Mann, Oliveira and Verwer/Hundsdorfer/ Sommeijer. A convergence concept for weak solutions of certain flow problems including entropy conditions is introduced by Ansorge. Some further contributions deal with finite element and finite difference methods for singularly perturbed convection-diffusion problems (Groen/ Veldhuizen, Stoyan. Tobiska).

The concluding third part contains applications of differential equations and of corresponding solution methods to the numerical modelling of real-world problems arising in different fields of natural sciences. Models involving ordinary differential equations are discussed by Bohl (transport of information in cells). Denk/Rentrop (simulation of electric circuits by the software package SPICE), Führer/Leimkuhler, Rösch/Kretzschmar (mechanical motion with restrictions), and Großmann/Juggi (heart physiology). The numerical determination of invariant tori for reaction. diffusion equations is investigated in a paper by Holodniok/Kubicek/Marek, thematically close to this topic is the note by Veldhuizen from Part 1 on the Josephson equation. Particular problems of fluid modelling (Abia/Sanz-Serna), models for chemical kinetics (Erhardt/Klusacek), solitons (Ortiz) and electrical field problems (Lucht/ Radke) are treated using partial differential equations.

Concluding remark after this short survey of the papers included into the proceedings of NUMDIFF-5: NUMDIFF- 6 will be held in Halle in September 1992 ! 